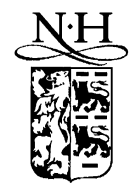

ELSEVIER

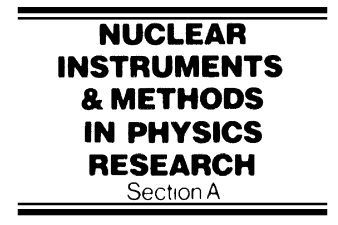

www.elsevier.nl/locate/nima

\title{
Modelling of an IR scintillation counter
}

\author{
M.M.F.R. Fraga ${ }^{a, b}, *$ F.A.F. Fraga ${ }^{a, b}$, A.J.P.L. Policarpo ${ }^{a, b}$ \\ ${ }^{a}$ LIP - Laboratório de Instrumentação e Física Experimental de Partículas, Delegação de Coimbra, 3004-516 Coimbra, Portugal \\ ${ }^{\mathrm{b}}$ Departamento de Física, Universidade de Coimbra, 3004-516 Coimbra, Portugal
}

\begin{abstract}
A systematic study of the excitation and de-excitation mechanisms in ternary gas mixtures $\mathrm{Ar}+\mathrm{CO}_{2}+\mathrm{N}_{2}$ is presented regarding the possibility of developing a proportional scintillation counter based on the detection of the infrared molecular emissions associated with the lowest vibrational states of molecules. The use of visible or near-infrared photons $(\lambda<1 \mu \mathrm{m})$ for applications like imaging and quality control of microstructure detectors has been reported. In view of these applications we analyse the processes leading to near-infrared emissions in pure argon and give an estimation of the number of photons emitted per electron, at several pressures, as a function of the charge gain. (C) 2000 Elsevier Science B.V. All rights reserved.
\end{abstract}

PACS: 29.40.Cs; 29.40.Mc

Keywords: Gaseous scintillators; Infrared emissions

\section{Introduction}

Most studies on gas scintillation proportional counters have been performed with pure rare gases or rare gas mixtures, taking profit of their high scintillation efficiency in electric fields below the threshold for charge multiplication [1]. In this case, the secondary scintillation emitted by Ar, Kr and Xe lies mainly in the VUV region and the total number of photons emitted is very much dependent on the purity of the gas, since the quenching of the atomic excited states by impurity molecules is very effective.

If the radiating states are low-energy states, like the low vibrational states of the ground state of

\footnotetext{
* Corresponding author.

E-mail address: margarida@lipc.fis.uc.pt (M.M.F.R. Fraga)
}

molecules, a large improvement in the number of excitations, up to about two orders of magnitude, can be obtained. To establish whether the detection of IR photons can be used to improve the performance of the scintillation counter (e.g., larger photon outputs and an improved energy resolution), as pointed out by Carugno [2], a detailed study of the excitation and de-excitation mechanisms involved is needed. We estimated the number of photons emitted per one electron drifting along $1 \mathrm{~cm}$, at normal pressure, in several $\mathrm{Ar}+\mathrm{CO}_{2}+\mathrm{N}_{2}$ mixtures (4.3 $\mu \mathrm{m} \mathrm{CO}_{2}$ band). $\mathrm{We}$ will show that, even in the mixtures where excitation efficiency is very high and the reabsorption of the $4.3 \mu \mathrm{m}$ photons is not very important, the number of emitted photons is considerably lower than in pure rare gases.

In parallel with these studies, we have been considering the use of visible or near-infrared (NIR) 
photons for applications like imaging and quality control of microstructure detectors, where scintillation efficiency is not very important. Indeed, it has been shown recently that a fair amount of light can be emitted from microstructures like MSGCs, MGCs and GEMs operated at moderate avalanche gain in mixtures containing noble gases as the main component or even pure argon [3]. In fact, in pure rare gases, if the electric field is increased above the charge multiplication threshold, the electrons gain high enough kinetic energy to excite the optical states and atomic lines, in the visible and/or near infrared regions, are observed [4,5]. It was proven that such scintillation light is very useful for identifying defects of the microstructures, as its yield is strongly correlated with the local electric fields seen by the drifting electrons. On the other hand, the readout of the GEM detector scintillation by means of an appropriate CCD constitutes, thanks to the fine structure of the GEM holes, a simple high-resolution position sensitive detector [6] convenient, for example, for imageology.

In this work, we present an analysis of the processes leading to NIR emissions in pure argon and an estimation of the number of photons emitted per primary electron as a function of the charge gain, for several pressures. Although this number is considerably lower than the number of VUV photons emitted in identical conditions, in pure rare gases, it may still be advantageous to use near-infrared photons if these can be handled in an easier or cheaper way.

\section{Infrared emissions in $\mathrm{Ar}+\mathrm{CO}_{2}+\mathrm{N}_{2}$ mixtures}

The lowest vibrational states of the ground state of the $\mathrm{CO}_{2}$ molecule are represented schematically in Fig. 1. As shown, the $\left(0,0^{\circ}, 1\right)$ state can decay to the $\left(1,0^{\circ}, 0\right)$ state $(10.6 \mu \mathrm{m}$ band $)$, to the $\left(0,2^{\circ}, 0\right)$ state $\left(9.6 \mu \mathrm{m}\right.$ band) and to the $\left(0,0^{\circ}, 0\right)$ state $(4.3 \mu \mathrm{m}$ band $)$, with transition probabilities of $0.35,0.2$ and $450.4 \mathrm{~s}^{-1}$ [7], respectively. The first two bands will be neglected due to their low transition probability.

One of the requirements to achieve a high efficiency is that the field energy should go mainly towards the excitation of the emitting state. Selective excitation of the $\left(0,0^{\circ}, 1\right)$ state is usually im-

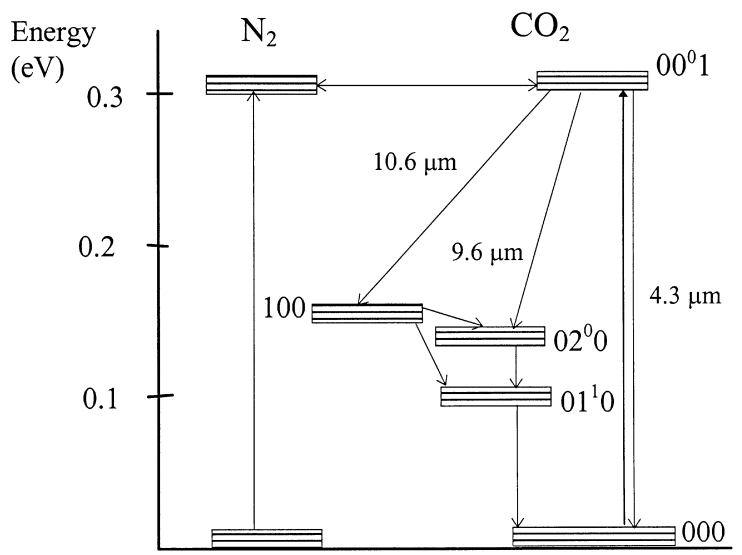

Fig. 1. Partial energy level diagram of $\mathrm{CO}_{2}$.

proved by adding nitrogen $[8,9]$. In fact, the $\left(0,0^{\circ}, 1\right)$ vibrational state of the $\mathrm{CO}_{2}$ molecule is nearly energy resonant with the vibrational state $v=1$ of the ground state of $\mathrm{N}_{2}$ (see Fig. 1) resulting in a very efficient energy transfer process. In addition, the excitation of this state by electron impact is also very efficient and it cannot decay through dipole radiation. Finally, the higher vibrational excited states $(v>1)$ of the electronic ground state of $\mathrm{N}_{2}$ are also nearly resonant with the corresponding $\mathrm{CO}_{2}$ levels (up to $00^{\circ} 4$ ), which are strongly coupled by collisions and decay very fast to the $\left(0,0^{\circ}, 1\right)$ state. For radiation detection purposes it is recommended to use a third gas as detection medium, as for example, argon or xenon. To avoid re-absorption of the $4.3 \mu \mathrm{m}$ band photons by $\mathrm{CO}_{2}$ molecules a low $\mathrm{CO}_{2}$ partial pressure is required.

The electron excitation coefficients were calculated either using a Boltzmann code, developed by Ségur and his group [10], or a Monte Carlo simulation. The Monte Carlo simulation code was developed for a parallel plane geometry using the null collision technique [11], and pre-calculated collision probabilities. Single electrons are followed up to the anode, taken as a perfect absorber. All the collisions were considered to be isotropic but the anisotropy of the elastic collisions was taken into account by replacing the total elastic cross-section by the momentum transfer cross-section. A good agreement between Monte Carlo and Boltzmann 


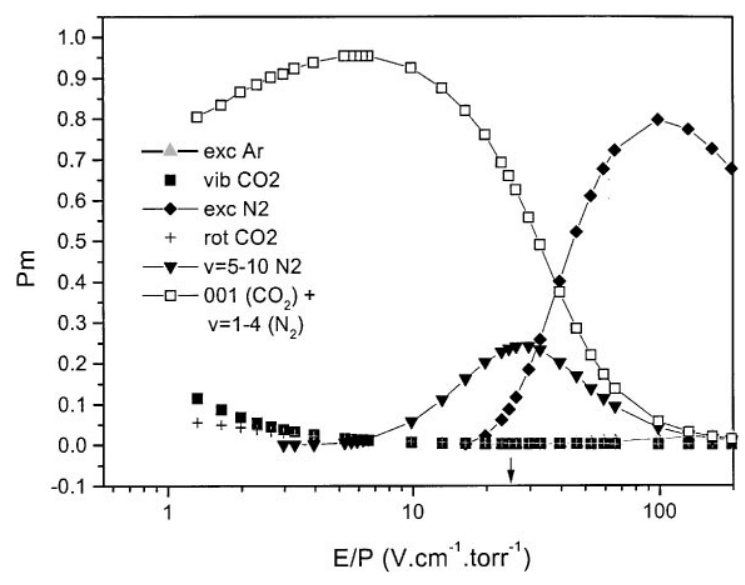

Fig. 2. Fractional power lost in several types of electron inelastic collisions as a function of the reduced electric field $(T=293 \mathrm{~K})$ in a nitrogen rich mixture $\left(10 \% \mathrm{Ar}+2 \% \mathrm{CO}_{2}+\right.$ $88 \% \mathrm{~N}_{2}$ ). The arrow indicates the threshold for charge multiplication.

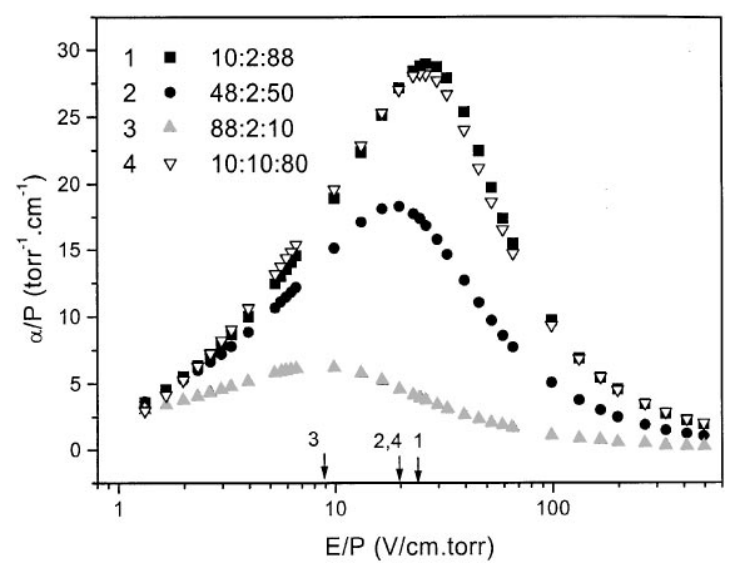

Fig. 3. Reduced excitation coefficients for the levels relevant to the emission of the $4.3 \mu \mathrm{m}$ band as a function of the reduced electric field, in some $\mathrm{Ar}+\mathrm{CO}_{2}+\mathrm{N}_{2}$ mixtures. The arrows indicate the charge multiplication thresholds for each mixture.

results was obtained and the Monte Carlo simulation was only used when information about the fluctuation on the number of excitations was needed.

In Fig. 2, the fractional power lost in inelastic collisions is plotted as a function of the reduced electric field for one of these mixtures showing that a high excitation efficiency of the relevant states $\left(00^{\circ} 1\right.$ of $\mathrm{CO}_{2}$ and $v=1-4$ of $\left.\mathrm{N}_{2}\right)$ is obtained. The electron excitation coefficient of these states increases with increasing concentration of $\mathrm{N}_{2}$ (see Fig. 3). In this plot, the population of each level by collisional de-activation or cascades from upper ones is not taken into account.

The kinetic scheme for the de-excitation of the $\mathrm{CO}_{2}\left(0,0^{\circ}, 1\right)$ level includes its radiative decay, the energy transfer process to the $\mathrm{N}_{2}(v=1)$ vibrational state and its de-excitation by two body collisions with species (Ar atoms, $\mathrm{CO}_{2}$ and $\mathrm{N}_{2}$ molecules) on their ground states $[12,13]$. The de-excitation of the $\mathrm{N}_{2}(v=1)$ level includes the energy transfer to the $\mathrm{CO}_{2}\left(0,0^{\circ}, 1\right)$ level and collisional de-activation by nitrogen molecules on their ground states [12]. The upper vibrational states of $\mathrm{N}_{2}$ also de-excite either by an energy transfer mechanism to the corresponding resonant $\mathrm{CO}_{2}$ $\left(0,0^{\circ}, n\right)$ levels or by two-body collisions to a lower vibrational state [14]. As these processes are all considered to be very fast, we can assume, as a reasonable approximation, that each $\mathrm{N}_{2}(v=k$, $k=2-4)$ state is equivalent to $k$ times $\mathrm{N}_{2}(v=1)$ levels. The light intensity shows then a single exponential decay law, with time constants of the order of a few hundreds of microseconds for normal pressures. In a stationary regime, the fraction of excitations which result in light emission decreases linearly with the pressure resulting in a non-dependence of the light intensity on the pressure. The mean number $\langle N\rangle$ of excitations per primary electron resulting in radiative decays is shown in Fig. 4. The ratios between the full-widths at half-maximum of the distributions, as given by the Monte Carlo calculations, and the mean values are also given in Fig. 4. These full-widths at half-maximum do not take into account uncertainties on crosssections or on rate coefficients.

Finally, it is important to consider the fraction of the $4.3 \mu \mathrm{m}$ band which is transmitted through the gas. This clearly depends on the geometry of the chamber. To give an idea of the effect of reabsorption of these photons by $\mathrm{CO}_{2}$ we take the results of Bulos and Phelps [12], who calculated the transmission factor for $\mathrm{CO}_{2} / \mathrm{N}_{2}$ mixtures. According to their results the transmission factor depends on the 


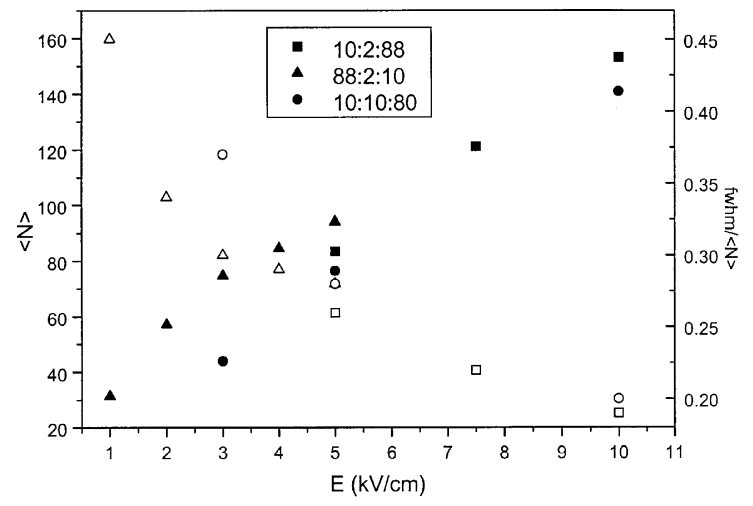

Fig. 4. Mean number of photons (solid symbols) emitted per electron per $\mathrm{cm}$ at 760 Torr and at $293 \mathrm{~K}$, as a function of the applied electric field, in three $\mathrm{Ar}+\mathrm{CO}_{2}+\mathrm{N}_{2}$ mixtures. The reabsorption of the $4.3 \mu \mathrm{m}$ band is not taken into account. The ratios between the full-widths at half-maximum and the mean values are also shown (open symbols). Fluctuations associated with the number of photons produced per electron do not take into account uncertainties on cross-sections or on rate coefficients.

Table 1

Transmission factor for two $\mathrm{CO}_{2}$ concentrations and two absorption lengths

\begin{tabular}{lll}
\hline & $r=0.5 \mathrm{~cm}$ & $r=5.0 \mathrm{~cm}$ \\
\hline $2 \% \mathrm{CO}_{2}$ & 0.54 & 0.17 \\
$10 \% \mathrm{CO}_{2}$ & 0.24 & 0.08 \\
\hline
\end{tabular}

relative concentration of $\mathrm{CO}_{2}$ and on the distance between the point of production and the window. Assuming that the photons are all emitted at the centre of a sphere of radius $r$, we estimate transmission factors as given in Table 1 .

The imprisonment of these photons by $\mathrm{CO}_{2}$ molecules does not affect much the lifetime of the emitting state because this is mainly determined by collisional processes.

The use of xenon instead of argon will not contribute to a significant increase of the fraction of radiative decays because the rate constant, $k_{\mathrm{Xe}}$, for the deactivation of $\mathrm{CO}_{2}\left(00^{\circ} 1\right)$ by two-body collisions with xenon atoms on their ground state is only slightly lower than $k_{\mathrm{Ar}}$ [13].

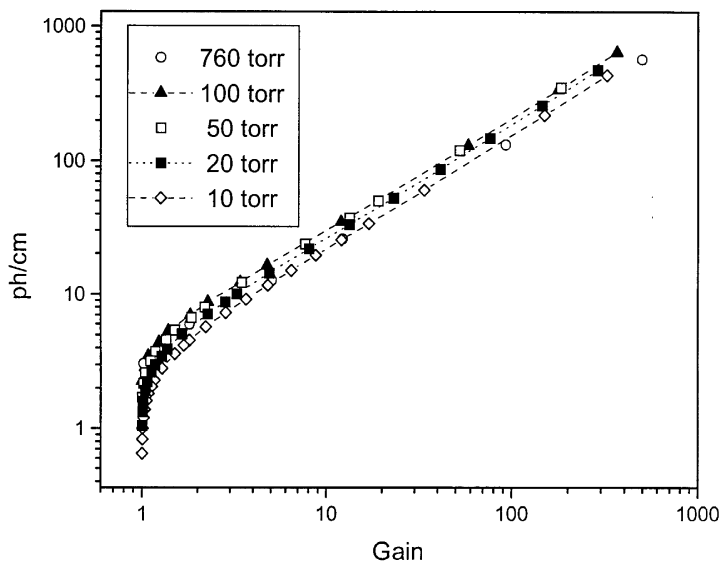

Fig. 5. Total number of near infrared photons $(\lambda<1 \mu \mathrm{m})$ emitted in $1 \mathrm{~cm}$ of argon as a function of the charge gain, for several pressures, for one primary electron.

\section{Near-infrared emission in pure argon}

The near-infrared emission spectrum of pure argon consists essentially of the atomic lines due to transitions between the atomic states of the $\mathrm{Ar}$ $\left(3 p^{5} 4 p\right)$ and $\operatorname{Ar}\left(3 p^{5} 4 s\right)$ configurations. In the wavelength region between 1 and $2 \mu \mathrm{m}$, other atomic lines resulting from transitions from upper states (e.g., from $\operatorname{Ar}\left(3 p^{5} 5 s\right)$ and $\left(3 p^{5} 3 d\right)$ levels) can also be present, but these are already out of the spectral range of conventional CCDs [3].

In the present work, we calculated the total number of photons corresponding to the $4 p \rightarrow 4 s$ transitions, taking into account not only their deactivation by collisions with argon atoms on their ground states [15], but also their population by cascades from upper states [16] (see Fig. 5). The population by collisional de-activation of upper states was not considered, due to the absence of experimental data. All the $\left(3 p^{5} 4 p\right)$ levels were considered as one level with mean lifetime of $30 \mathrm{~ns}$ [15] and mean quenching rate for intermultiplet collisions of $10^{6}$ Torr $^{-1} \mathrm{~s}^{-1}$. The $5 \mathrm{~s}$ and $3 \mathrm{~d}$ states were considered also as one state with a mean quenching rate similar to the one used for the $4 p$ levels [16]. 


\section{Conclusion}

The fluctuations associated with the number of photons produced per electron in $\mathrm{Ar}+\mathrm{CO}_{2}+\mathrm{N}_{2}$ mixtures are low compared to fluctuations associated with other quantities involved in radiation detection (namely, the Fano factor). However, the use of these particular mixtures in proportional scintillation counters, operating in the pulse mode, does not seem to be appropriate due to the long lifetime of the emitting state and the low number of photons emitted per electron. Nevertheless, there is the possibility of working in the current mode and of using other field geometries and/or other gas mixtures. Further studies on the IR emissions of atomic and molecular gases are under way.

In what concerns the use of NIR emissions for imaging and quality control applications, the calculations, supported by experimental evidence, have shown that a comfortable number of photons is emitted even in the presence of a small admixture of $\mathrm{CO}_{2}$. These photons match the spectral response of standard CCD cameras and the gas can be easily kept in a flow regime. The study of rare gas mixtures, such as neon-argon mixtures and argon plus a small amount of xenon are under investigation.

\section{Acknowledgements}

Acknowledgements are due to Dr. P. Ségur, from the Centre de Physique Atomique, Université Paul
Sabatier, Toulouse, for providing us with his Boltzmann code. The electron impact cross-section data were obtained from Dr. P. Ségur and from Dr. J. Loureiro from Centro de Electrodinâmica, IST, Lisbon. This work was supported by contract CERN/P/FIS/1198/98 with the Portuguese FCT.

\section{References}

[1] A.J.P.L. Policarpo, Phys. Scripta 23 (1981) 539

[2] G. Carugno, Nucl. Instr. and Meth. A 419 (1998) 617.

[3] F.A.F. Fraga et al., Nucl. Instr. and Meth. A, in press.

[4] P.E. Thiess, G.H. Miley, IEEE Trans. Nucl. Sci. NS-21 (1974) 125.

[5] P. Lindblom, O. Solin, Nucl. Instr. and Meth. A 268 (1988) 204

[6] F. Fraga et al., Nucl. Instr. and Meth. A 422 (2000) 417

[7] A.A. Radzig, B.M. Smirnov, Springer Series in Chem. Phys. Vol. 43, Springer Berlin, 1985.

[8] O. Svelto, Principles of Lasers, Plenum Press, New York.

[9] W.J. Witteman, The $\mathrm{CO}_{2}$ Laser, Springer Series in Optical Sciences, Vol. 53, Springer, Berlin, (1987).

[10] P. Ségur et al., Transp. Theor. Stat. Phys. 15 (1986) 705.

[11] H.R. Skullerud, J. Phys. D 1 (1968) 1567.

[12] B.R. Bulos, A.V. Phelps, Phys. Rev. A 14 (1976) 615.

[13] J.T. Yardley, C.B. Moore, J. Chem. Phys. 46 (1967) 4491.

[14] C.K.N. Patel, J. Chim. Phys. 64 (1967) 82.

[15] R.S.F. Chang, D.W. Setser, J. Chem. Phys. 69 (1978) 3885.

[16] J.W. Keto, C.-Yu Kuo, J. Chem. Phys. 74 (1981) 6188. 